\title{
LIMIT-PRESERVING EMBEDDINGS OF PARTIALLY ORDERED SETS IN DIRECTED SETS
}

\section{J. R. ISBELL AND HERMAN RUBIN ${ }^{1}$}

Let $(A,>)$ be a partially ordered set. Then we say [3] that a function $f$ on $A$ decides for $S$ (on $A$ ) if $\{\alpha \mid f(\alpha) \in S\}$ contains a cofinal residual subset of $A$, i.e., if $\gamma \in A$, there is a $\beta \geqq \gamma$ such that for all $\alpha \geqq \beta, f(\alpha) \in S$. Clearly the intersection of any two cofinal residual sets is cofinal residual. Ginsburg [2] showed that any partially ordered set $(A,>)$ can be embedded in an everywhere branching set $(B,>)$ in such a manner that the "natural" extension of $f$ to $B$ decides for the same sets as $f$ does. Day [1] has an embedding in a directed set preserving decision, but possibly creating new decisions, even turning a nonconvergent function into a convergent one. We show here that the embedding can be made precise. First, let us notice that

Lemma. Let $(A,>)$ and $(B,>)$ be partially ordered sets and let $\phi$ map $B$ into $A$. If

(i) for every $T$ cofinal residual in $A, \phi^{-1} T$ is cofinal residual in $B$, and

(ii) for every $U$ cofinal residual in $B, \phi U$ is cofinal residual in $A$, then for every $f$ and $S, f$ decides for $S$ on $A$ if and only if $f \phi$ decides for $S$ on $B$.

We may assume (by a trivial preliminary embedding) that the given set $A$ has no least cofinal residual set, i.e., the set of maximal elements is not cofinal. Let $B$ be the set of all pairs $(T, \alpha)$ such that $\alpha \in T$ and $T$ is cofinal residual in $A$. We define $\left(T_{1}, \alpha_{1}\right)>\left(T_{2}, \alpha_{2}\right)$ if $T_{1} \subset T_{2}$ or $T_{1}=T_{2}$ and $\alpha_{1}>\alpha_{2}$. We define $\phi(T, \alpha)=\alpha$. Thus we see that $(B,>)$ is directed and therefore cofinal residual reduces to residual. Now if we replace $(A, \alpha)$ by $\alpha$, we see that

THEOREM. Every partially ordered set $(A,>)$ can be embedded in a directed set $(B,>)$ in such a manner that for any function $f$ on $A$, the "natural" extension of $f$ to $B$ decides for precisely those sets for which $f$ decides.

\section{REFERENCES}

1. Mahlon Day, Oriented systems, Duke Math. J. vol. 11 (1944) pp. 201-212.

Received by the editors August 12, 1954 and, in revised form, September 11, 1955.

${ }^{1}$ Authors now (1) at the Institute for Advanced Study and (2) at the University of Oregon. 
2. Seymour Ginsburg, Real-valued functions on partially ordered sets, Proc. Amer. Math. Soc. vol. 4 (1953) pp. 356-359.

3. John Tukey, Convergence and uniformity in topology, Princeton, 1940.

Princeton University and

STANFORD UNIVERSITY

\section{THE ZEROS OF CERTAIN SINE-LIKE INTEGRALS}

A. E. LIVINGSTON AND LEE LORCH

We establish here the monotonic character of the zeros (modulo 1) of

$$
\int_{x}^{\infty} \frac{f(t)}{t} d t, \quad x>0,
$$

where $f(t)$ satisfies the conditions

(C1). $f(t) \geqq 0$ for $0 \leqq t<1$;

(C2). $f(t) \not \equiv 0$ on any subinterval of $0 \leqq t<1$;

(C3). $f(t+n)=(-1)^{n} f(t)$ for $n=1,2,3, \cdots$;

(C4). $f(t) / t$ is Lebesgue integrable on $0 \leqq t \leqq 1$.

It is clear that these conditions imply that the integral (1) has precisely one zero, say $z_{n}$, in the interval $n<x<n+1$.

Let $C$ be defined (uniquely) by the conditions

$$
2 \int_{0}^{C} f(t) d t=\int_{0}^{1} f(t) d t, \quad 0<C<1 .
$$

Now,

(A) $z_{n}-n \geqq C$ for $n=0,1,2, \cdots$,

(B) $z_{n}-n \rightarrow C$ as $n \rightarrow \infty$,

as was shown in [2], even more generally, with the factor $1 / t$ of $f(t)$ in (1) replaced by a function denoted there by $g(t)$ of which $1 / t$ is a special case. When $f(t)=\sin \pi t$, the sequence $\left\{z_{n}-n\right\}_{0}^{\infty}$ is decreasing, as Harry Pollard has shown, and I. I. Hirschman has observed that Pollard's proof applies equally well to the zeros of

$$
\int_{x}^{\infty} g(t) \sin \pi t d t
$$

Presented to the Society June 18, 1955; received by the editors December 13 . 1954 and, in revised form, October 11, 1955. 